\title{
Tratamiento moderno de la osteocondrosis en equinos ¿qué podemos ofrecer?
}

\section{Roberto Estrada McDermott ${ }^{1} \otimes$}

1 Cátedra de Cirugía de Especies Mayores y Hospital Equino, Escuela de Medicina Veterinaria, Universidad Nacional. Email: roberto.estrada.mcdermott@una.cr

La osteocondrosis (OCD) es una de las enfermedades del desarrollo más comunes en el equino. En general, se produce un defecto focal en la osificación endocondral que causa la separación de un fragmento osteoncondral dentro de la articulación. Esta es una patología multifactorial, pero está relacionada, en gran medida, con la transmisión genética y los desbalances nutricionales. A pesar de programas de selección en el nivel internacional, esta enfermedad sigue estando presente alrededor del mundo y es una causa importante de desecho de animales causado por los cambios osteoartríticos secundarios y claudicación. No obstante, se sabe que el pronóstico de esta enfermedad depende en gran medida del tamaño y localización del fragmento, un diagnóstico temprano y un tratamiento quirúrgico mínimamente invasivo. Modernamente se recomienda combinar el diagnóstico radiológico con ultrasonografía, ya que se ha demostrado que la segunda técnica es más sensible para la detección de fragmentos. Además, el ultrasonido permite localizar en tiempo real los fragmentos y evaluar los tejidos blandos articulares. Desde el punto de vista terapéutico, es de vital importancia tener en cuenta que las terapias intraarticulares no son más que paliativas y no permiten solucionar el problema, esta línea de terapias cumple un rol importante en el contexto de que los fragmentos sean removidos de la articulación. Se ha demostrado que la remoción de los fragmentos por medio de técnicas mínimamente invasivas presentan una menor morbilidad y mucho mejores tasas de éxito que las técnicas más invasivas. El objetivo de esta presentación es dar a conocer datos actuales sobre la epidemiología y pronóstico de las presentaciones más comunes de esta enfermedad, además de describir una metodología moderna para el diagnóstico y tratamiento de esta patología.

凶 Autor para correspondencia Roberto Estrada McDermott: roberto.estrada.mcdermott@una.cr 